\title{
In-Situ Calibration of UFFO/Lomonosov for Observation of GRBs
}

S. Jeong ${ }^{1,2}$, I. H. Park ${ }^{1 *}$, V. Bogomolov ${ }^{3}$, S. Brandt ${ }^{4}$, C. Budtz-Jørgensen ${ }^{4}$, A.J. Castro-Tirado $^{2,5}$, S.-H. Chang ${ }^{6}$, Y.Y. Chang ${ }^{6}$, P. Chen ${ }^{7,8}$, C.-R. Chen ${ }^{6}$, C.-W. Chen $^{7}$, H. S. Choi ${ }^{9}$, P. Connell ${ }^{10}$, C. Eyles ${ }^{10}$, G. Gaikov ${ }^{1}$, G. Garipov ${ }^{3}$, J.-J. Huang $^{7}$, M.-H. A. Huang ${ }^{11}$, H.M. Jeong ${ }^{1}$, J.E. Kim ${ }^{1}$, M.B. Kim ${ }^{1}$, S.-W. Kim ${ }^{12}$, H.K. Lee' ${ }^{13}$, J. Lee', H. Lim, C.-Y. Lin', T.-C. Liu', J.W. Nam ${ }^{7,8}$, M.I. Panasyuk $^{3}$, V. Petrov ${ }^{3}$, V. Reglero ${ }^{10}$, J. Ripa ${ }^{7,8}$, J.M. Rodrigo ${ }^{10}$, S. Svertilov ${ }^{3}$, M.Z. Wang ${ }^{7}$, I. Yashin ${ }^{3}$

${ }^{1}$ Department of Physics, Sungkyunkwan University, 2066, Seobu-ro, Jangan-gu, Suwon, Gyeonggi-do, 16419, Korea

${ }^{2}$ Instituto de Astrofisica de Andalucia (IAA-CSIC), P. O. Box 03004, E-18080 Granada, Spain

${ }^{3}$ Skobeltsyn Institute of Nuclear Physics of Lomonosov, Moscow State University, Leninskie Gory, Moscow, 119234, Russia

${ }^{4}$ National Space Institute, Astrophysics, Technical University of Denmark, DK-2800, Kongens, Lyngby, Denmark

${ }^{5}$ Departamento de Ingeniería de Sistemas y Automática, Universidad de Málaga, Spain

${ }^{6}$ National Space Organization, 9 Prosperity $1^{\text {st }}$ Road, Hsinchu Science Park, HsinChu, 30078, Taiwan

${ }^{7}$ Department of Physics, National Taiwan University, 1 Roosevelt Road, Taipei, 10617, Taiwan

${ }^{8}$ Leung Center for Cosmology and Particle Astrophysics, National Taiwan University, 1 Roosevelt Road, Taipei, 10617, Taiwan

${ }^{9}$ Korea Institute of Industrial Technology, 89 Yangdaegiro-gil, Seobuk-gu, Cheonan-si, Chungcheongnamdo, 331-822, Korea

${ }^{10}$ GACE, Edif. de Centros de Investigacion, Universidad de Valencia, Burjassot, E-46100

Valencia, Spain

${ }^{11}$ Department of Energy Engineering, National United University, 2, Lienda Rd., 36063 Miaoli, Taiwan

${ }^{12}$ Department of Astronomy, Yonsei University, 134 Shinchon-dong, Seoul 120-749, Korea

${ }^{13}$ Camera Development Group, Mobile Communication Business Division, SAMSUNG Electronics Co.Ltd., Korea

E-mail: soominjeong@gmail.com

The UFFO/Lomonosov has been successfully launched into Sun synchronous orbit and is operational through tests and calibrations since its launch on Apr. 28, 2016. As a pathfinder of UFFO, it will be the first space instrument to use a fast slewing mirror which reduce the trigger latency of optical telescope, less than a second, to explore early time domain of GRB evolution. In this article, we will report in detail the first mission, UFFO/Lomonosov, for the rapid response to observe early photons from GRBs in orbit.

35th International Cosmic Ray Conference - ICRC2017

10-20 July, 2017

Bexco, Busan, Korea

*Corresponding author

c Copyright owned by the author(s) under the terms of the Creative Commons 


\section{Introduction}

Thanks to Swift (Gehrels et al., 2004) and ground robotic telescopes, hundreds of GRB afterglows have been measured in X-ray and optical regime, they reveals many intriguing features in X-ray which are not expected by standard fireball, such as complex light curves of X-ray afterglows. Though they look have common features, but optical light curve are rather simple than X-ray afterglow. To understand them, we need a rapid telescope which could respond promptly in optical regime to explore the mechanism of material ejects from burst and its environments.

The Ultra-Fast Flash Observatory (UFFO) was proposed to measure the UV/optical counterpart on second time scales (Park et al., 2013) after the gamma ray trigger from GRBs by introducing very fast pointing of the narrow-FOV UV/optical telescope using a fast slewing mirror or mirror array (Park et al., 2009). It provides a reliable method to lower the current response limit by one to two orders of magnitude in order to obtain a large statistically meaningful sample of GRB light curves including the rise phase for the first time. In this paper, general description on UFFO/Lomonosov to catch the early photons from GRBs will be following in next session and its in-situ calibration status will be discussed briefly.

\section{Approach to observe the early photons in UFFO/Lomonosov}

The payload of UFFO/Lomonosov is composed of two telescopes: a trigger and localizing telescope in X-ray, UBAT, and a narrow-FOV fast optical telescope, SMT, which is key idea of UFFO. Once UBAT gives a trigger to SMT, SMT is enable to slew any regimes with a second over its $70 \times 70$ degree FOV (Park, 2004; Park et al., 2008; Lee et al., 2012). The SMT (Jeong et al., 2013) is a 10$\mathrm{cm}$ diameter aperture modified Ritchey-Chretien telescope equipped with a reflector of $15-\mathrm{cm}$ diameter slewing mirror and UBAT adopts a coded mask technique and used advanced FPGA with a lower number of detector channels. We reduce the trigger latency significantly, the required time of trigger decision, including computation speed for the trigger processing, less than a second and thus an order of magnitude faster than that of the Swift. The FOV of UBAT is $70.4 \times 70.4 \mathrm{deg}^{2}$ for half coded, which overlaps the full coverage of the SMT, $70 \times 70 \mathrm{deg}^{2}$. 


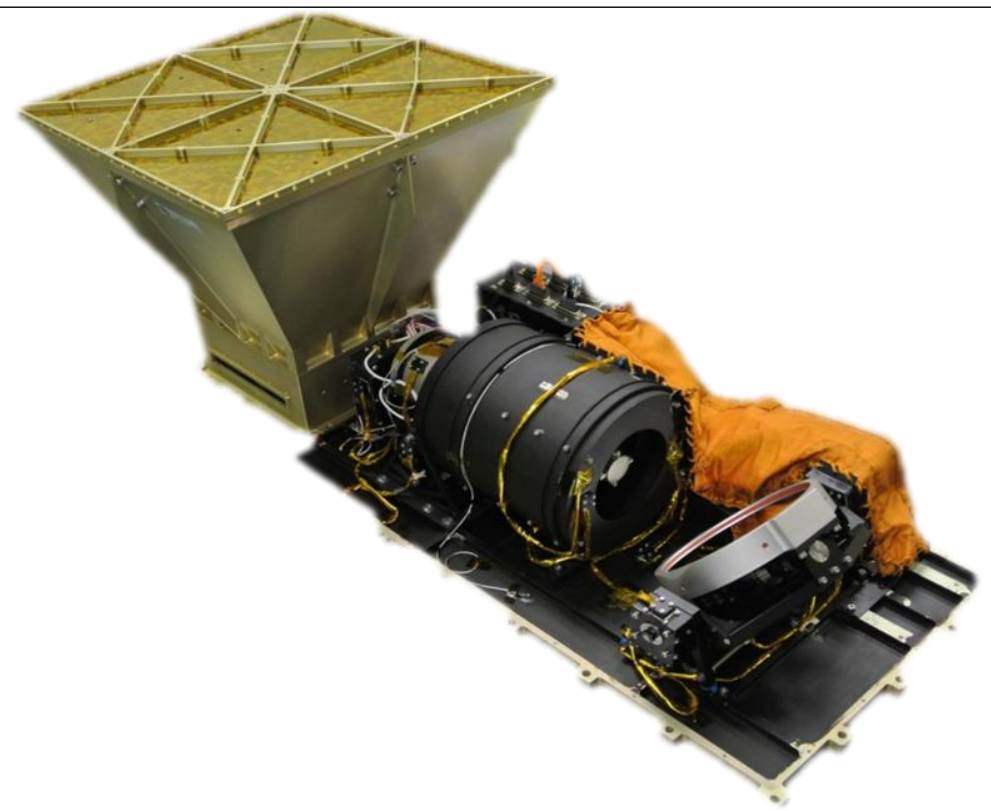

Fig. 1 The flight model of UFFO/Lomonosov, Gold part is X-ray telescope of UFFO Burst Alert \& Trigger Telescope and the other is optical Slewing Mirror Telescope.

\section{In-orbit calibration of UFFO/Lomonosov}

Since the launch on Apr 28, 2016, UFFO/Lomonosov has been tested and calibrated till the end of Nov. 2016 for which the UFFO instrument was powered on 20 times, each time orbiting once and running about 25 mins except dawn and dusk. Several sets of parameters designed for X-ray trigger and alignment of the slewing mirror for a given direction were examined. Figure 2 shows the behavior of UFFO power consumption in terms of currents measured from satellite sensor. The UFFO was found to run as designed, i.e., after initialization of the instrument, trigger occurred which enabled data taking of SMT and UBAT, followed by data transfer to the satellite. We tested such a run sequencing several times to find working as designed. In this calibration, we set the trigger threshold low enough on purpose to generate a false trigger from backgrounds.

Figures 3 shows a sky image obtained during calibration runs of the UFFO on Sep 2016. The SMT has $17.7^{\prime} \times 17.7^{\prime}$ field of view. The exposure time was $0.6 \mathrm{sec}$. The image center lies at RA= 22:46:46.56, Dec=-08:14:31.20. Comparing the USNO B catalogue, we found the upper limit of SMT to be about 19 magnitude for $100 \mathrm{sec}$ as considering correction effects. The value agrees with the designed. 


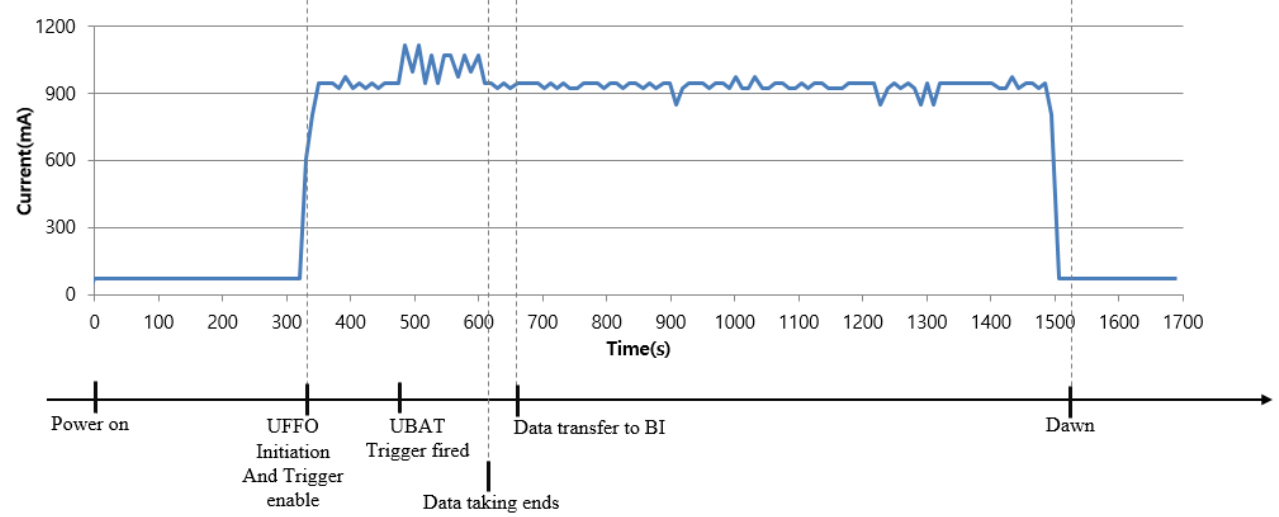

Fig. 2 The behavior of the UFFO instrument in terms of currents monitored by satellite. The running sequence was confirmed such that initialization of the instrument took $\sim 5 \mathrm{~min}$ and $36 \mathrm{sec}$, then data taking of SMT and UBAT started on trigger, and finally data transfer to the satellite was made.
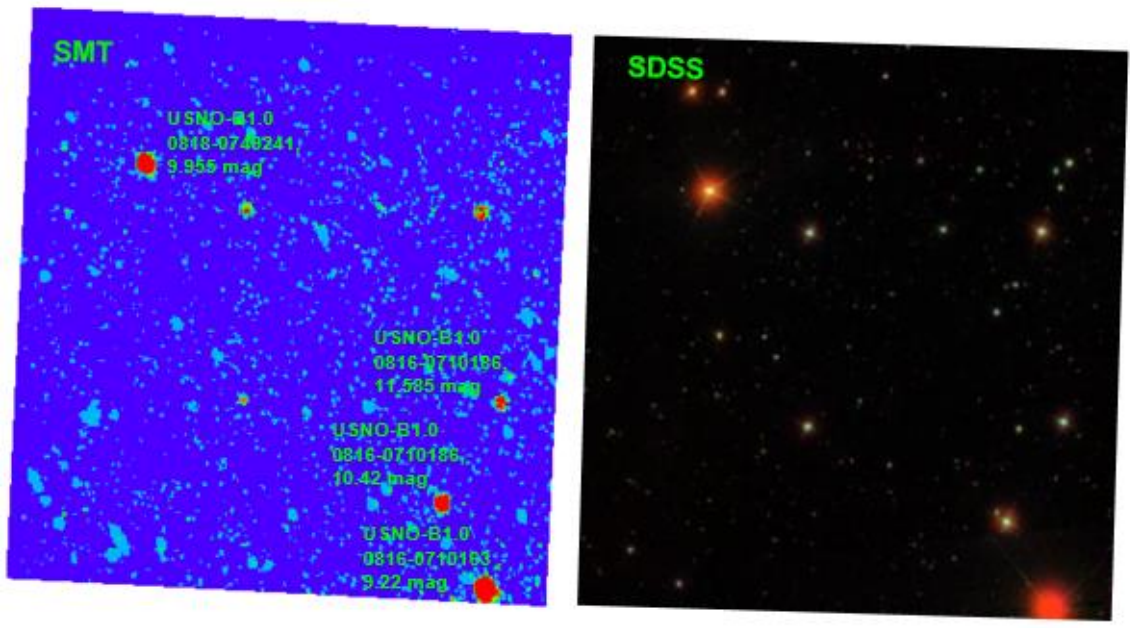

Fig. 3 The sky image of SMT observed on Sep. 8, 2016 during the SMT calibration on orbit. The image center lies at RA= 22:46:46.56, Dec=-08:14:31.20. Comparing the USNO B catalogue, we found the upper limit of SMT to be about 19 magnitude for $100 \mathrm{sec}$ as expected.

\section{Summary}

The payload of the UFFO (Ultra-Fast Flash Observatory)-pathfinder now onboard the Lomonosov spacecraft (hereafter UFFO/Lomonosov) is a dedicated instrument for the observation of GRBs. Its primary aim is to capture the early phase of the optical light curve, one of the least known aspects of GRBs. Fast response measurements of the optical emission of GRB will be made by a SMT, a key instrument of the payload, which will open a new frontier in transient studies by probing the early optical rise of GRBs with a response time in seconds for the first time. The SMT employs a rapidly slewing mirror to redirect the optical axis of the telescope to a GRB position prior determined by UBAT, the other onboard instrument, for the observation and imaging of X-rays. UFFO/Lomonosov was launched successfully from Vostochny, Russia on April 28, 2016, and will begin GRB 
observations after completion of functional checks of the Lomonosov spacecraft. From series of calibration, we confirmed its main concept, slewing mirror working and it can respond to initial photons within a couple of seconds after trigger, which improves on current optical response times by a couple of orders of magnitude and tracking the object for tens of seconds. The number of GRBs is expected to be 10 20 per year.

\section{References}

[1] N. Gehrels et al., The Swift Gamma-Ray Burst Mission, ApJ 611, 1005 (2004)

[2] I.H. Park et al., Ultra-Fast Flash Observatory for the observation of early photons from gamma-ray bursts. NJP 15023031 (2013)

[3] I.H. Park et al., The UFFO (Ultra-Fast Flash Observatory) Pathfinder, arXiv:0912.0773 (2009)

[4] I.H. Park, MEMS based Space Telescope for Extreme Energy Cosmic Rays Experiments. NuPhS 134, 196 (2004)

[5] J.H. Park et al., Obscura telescope with a MEMS micro mirror array for space observation of transient luminous phenomena or fast-moving objects, Opt. Express 1620249 (2008)

[6] J. Lee et al., A New Type of Space Telescope for Observation of Extreme Lightning Phenomena in the Upper Atmosphere. ITGRS, 50, 10, 3941 (2012)

[7] S. Jeong et al., Slewing Mirror Telescope Optics for the Early Observation of UV/optical Photons from Gamma-Ray Bursts, Opt. Express 21, 2, 2263 (2013) 